\title{
Interaction of cerebrovascular disease and contralateral carotid occlusion in prediction of shunt insertion during carotid endarterectomy
}

María J. Estruch-Pérez ${ }^{1}$, Angel Plaza-Martínez², Maria J. Hernández-Cádiz¹, Juan Soliveres-Ripoll², Cristina Solaz-Roldán', María M. Morales-Suarez-Varela³

\author{
${ }^{1}$ Anaesthesiology and Critical Care Department, Dr. Peset University Hospital, \\ Valencia, Spain \\ 2Vascular Surgery Department, Dr. Peset University Hospital, Valencia, Spain \\ 3Unit of Public Health and Environmental Care, Department of Preventive Medicine, \\ University of Valencia, Spain; Unit of Clinical Epidemiology, Dr. Peset University \\ Hospital, Valencia, Spain; and CIBER of Epidemiology and Public Health (CIBERESP), \\ Spain \\ Submitted: 11 March 2011 \\ Accepted: 24 July 2011
}

Arch Med Sci 2012; 8, 2: 236-243

DOI: 10.5114 /aoms.2012.28550

Copyright (c) 2012 Termedia \& Banach

\author{
Corresponding author: \\ María J. Estruch Pérez MD \\ Anaesthesiology and \\ Critical Care Department \\ Dr. Peset University Hospital \\ Avda. Gaspar Aguilar n 90 \\ 46017 Valencia (Spain) \\ Phone: +34654800851 \\ Fax: +3496.386.25.01 \\ E-mail: estruch_mar@gva.es
}

\begin{abstract}
Introduction: To assess the possible role and the interaction of cerebrovascular disease and vascular stenosis on the necessity of shunt insertion during carotid endarterectomy (CEA).

Material and methods: Eighty consecutive patients undergoing CEA under regional anaesthesia were prospectively enrolled. Patients were divided into two groups depending on whether they were shunted or not. The measured end-points were co-morbidities degree of contralateral and carotid stenosis and other intraand postoperative outstanding parameters. ANOVA, Student's $t$ and $\chi^{2}$ tests were used $(p<0.05)$. Variables differing significantly between groups and potential confounders were used in backward stepwise logistic regression to estimate the relative risk $(\mathrm{RR}, 95 \% \mathrm{Cl})$ of shunt. In addition Wald's test $(p<0.05)$ with and without adjustments for potential confounders was used with various different multivariate analysis models.

Results: Contralateral stenosis and cerebral vascular accidents (CVA) were more frequently observed in shunted patients. The RR for patients with contralateral stenosis $\geq 50 \%$ was $1.3(95 \% \mathrm{Cl} 1.0-1.5)$ and for patients with previous CVA was 1.2 (95\% Cl 1.0-1.4). For contralateral stenosis and CVA together the RR increased to 7.7 (95\% Cl 1.0-14.4). A model based on contralateral stenosis and CVA was found to be statistically significant $(p=0.003)$ for shunt $(R R=1.1$, $95 \% \mathrm{Cl} 1.0-2.1$ ). Relative excess risk due to interaction of both factors was 6.2 . Conclusions: The findings suggest that patients with contralateral stenosis $\geq 50 \%$ and previous CVA have a higher risk of requiring shunt use during CEA than patients with these risk factors separately.
\end{abstract}

Key words: carotid endarterectomy, shunted patients, cerebral ischaemia.

\section{Introduction}

Carotid endarterectomy (CEA) has become a well-established technique to prevent the risk of stroke in symptomatic and asymptomatic patients, as reviewed in recent trials [1, 2]. However, the long-term benefit must outweigh the perioperative risk (stroke and death). Most perioperative strokes 
are ischaemic and some are caused by low blood flow during the carotid clamping period. The insertion of an intraluminal shunt to minimize the risk of low flow and intraoperative stroke has been used. A recent Cochrane Review [3] of routine versus selective carotid artery shunting showed that the currently available data were too limited to support or refute the use of routine or selective carotid shunting in patients undergoing CEA under general anaesthesia. However, the use of a shunt makes the operation technically more demanding and carries potential complications such as embolism or damage to arterial walls. Shunt insertion has also been related to postoperative internal carotid artery (ICA) re-stenosis after CEA [4] and to subclinical neurocognitive dysfunction [5], although the literature also shows controversy. In this context, it would be an advantage to preoperatively predict which patient will need a shunt. Neuromonitoring methods easy to apply, such as bispectral index, have been investigated for monitoring of cerebral ischaemia to allow selective shunt insertion [6].

Factors such as moderate ipsilateral carotid stenosis, degree of contralateral stenosis or preoperative symptoms of cerebrovascular disease [7-15] have been associated with shunt placement due to cerebral ischaemia during CEA. These reports suggest shunting patients with the factors mentioned with poor strength of association that could improve with the combination of factors. With awake patients under regional anaesthesia it was possible for us to use a selective shunt while monitoring neurological status during surgery. With this approach, we undertook an observational prospective study to identify preoperative or intraoperative characteristics of the patients associated with neurological changes consistent with cerebral ischaemia which needed shunting. In particular, we assess the possible role and the interaction of cerebrovascular disease and vascular stenosis on the necessity of shunt insertion during CEA.

\section{Material and methods}

This prospective observational single-centre study was conducted in the vascular surgery department at Dr Peset Hospital, Valencia, Spain, from November 2005 to December 2007. The protocol was approved by the local ethics committee, which waived the requirement for informed consent as data were collected while care of patients conformed to standard procedures currently used in our institution. During a three-year period, all patients undergoing CEA $[1,2]$ were operated on under a regional anaesthesia protocol and they were invited to participate in the study. Patients allergic to regional anaesthetics, who refused regional anaesthesia, with cognitive limitation, anxiety, orthopnoea, a known language barrier or ASA $\checkmark$ were excluded from the protocol. Based on these criteria, 80 patients were included and, of these, only one was converted to general anaesthesia.

Perioperative management was standardized. For the preoperative selection of study patients the main tool used for extracranial screening, diagnosis and follow-up was Echo-Doppler testing in accordance with the consensus conference of the Society of Radiologists in ultrasound [16]. Other studies such as arteriography or magnetic resonance angiography were only used if doubts were found with echoDoppler testing about the stenosis percentage [1].

Standard instrumentation was used in the operating room: all patients were monitored using fivelead ECG, pulse oximetry, and invasive measurement of mean arterial pressure (MAP). An $18 \mathrm{G}$ intravenous cannula was placed in the forearm and intravenous premedication, either 1-2 mg of midazolam (MDZ) or $1 \mathrm{mg}$ MDZ and $50 \mu \mathrm{g}$ of fentanyl, was given 10 minutes before placing a cervical plexus block to provide anxiolysis, as deemed necessary by the anaesthetist. During the procedure, each patient received oxygen at $3 \mathrm{l} / \mathrm{min}$ via nasal prongs. For regional anaesthesia, $20 \mathrm{ml}$ of bupivacaine $0.375 \%$ was administered for the deep cervical plexus block following a modified version of the single-injection technique proposed by Winnie et al. [17] or using a three-injection technique at $C_{2}$, $\mathrm{C}_{3}$ and $\mathrm{C}_{4}$ as described by Moore [18]. Mepivacaine $2 \%$ (20 ml) was administered for the superficial cervical plexus block at the midpoint of the posterior border of the sternocleidomastoid muscle. If the patient noted pain during surgery, the surgeon administered supplementary $1 \%$ lidocaine directly around the carotid sheath. Also, $0.10 \mathrm{mg}$ of fentanyl was used in cases when additional sedation or pain relief was required in the course of the surgery. The MAP was maintained using vasodilators or volume expanders and catecholamines to maintain systolic blood pressure within the normal limits ( $\pm 20 \%$ of basal blood pressure values).

Carotid stenosis consists of removal of plaque through the open common carotid by means of a pre-jugular approach. Clamping of the internal and external carotid is necessary. Heparin (5000 U i.v.) was given before carotid cross-clamping. A clamping test was performed for 3 min to determine tolerance to low cerebral flow through the carotid artery. Cerebral ischaemia during clamping was assumed when neurological changes were detected and a Javid shunt was placed from the common carotid artery to the intact internal carotid artery. The carotid was closed directly or by means of a path. Heparin was reversed with protamine after surgery in some patients at the discretion of the surgeon. The patient was kept in the ICU for the first $24 \mathrm{~h}$ to allow close monitoring of blood pressure, neurological condition and drainage. Pre- and 
postoperative routine antiplatelet drug therapies (acetylsalicylic acid, clopidogrel) were not withheld and were given according to pre-existing prescriptions. In all patients the CEA was performed using the same method and by the same surgical team. Patients continued to take their routine medication until the morning of surgery.

\section{Data collection}

For each patient, the following data were collected: preoperative risk factors for morbidity and mortality (patient characteristics, smoking habit, main identified comorbidities); previous symptoms (cerebral vascular accident - CVA, transient ischaemic accident - TIA); American Society of Anesthesiology (ASA) classification, and preoperative medication; intra-postoperative data (degree of ipsilateral stenosis and contralateral carotid stenosis, duration of surgery and clamping, ICU and hospital stay, postoperative blood pressure, intrapostoperative antihypertensive therapy); and 30-day postoperative complications (death, neurological signs and symptoms (CVA, TIA), acute myocardial infarction (AMI), and other complications such as cranial nerve deficits and surgical haematoma). These data were prospectively entered into a database for later analysis.

Relevant previous symptoms were considered to be CVA or TIA, including amaurosis fugax as a symptom of carotid stenosis. Patients were considered to be asymptomatic if the aforementioned pathologies and the carotid stenosis had been detected following a full vascular assessment in the context of another vascular disease or as an incidental observation made during an examination for another non-vascular disease.

Cerebral ischaemia during clamping was assumed when neurological changes were detected. Specifically, the neurological changes considered to indicate cerebral ischaemia were as follows: focal neurological deficits (tested by requesting the patient to move each of their limbs and squeeze a toy); decreased consciousness [19], tested by assessing speech using the verbal component of the Glasgow Coma Scale; and dysarthria [19]. The period of carotid clamping used to record neurological changes during neurological monitoring was a 3-min clamping test. The need for Javid shunt placement was based on the occurrence of neurological changes during this 3-min clamping test and whether neurological deficits required conversion from regional to general anaesthesia prior to shunt insertion.

\section{Statistical analysis}

For data analysis, we divided a posteriori the 80 individuals into two groups according to shunt insertion during surgery: shunted patients $(n=12)$ and non-shunted patients $(n=68)$.
Quantitative results are presented as means and standard deviations (SD), minimum and maximum, while qualitative results are reported as absolute and relative (percentages) frequencies.

Statistical analysis was performed in two steps. The first step was a descriptive analysis with twoway repeated measures ANOVA for continuous variables and the $\chi^{2}$ test for proportions to compare differences between shunted and non-shunted groups; $p$-values less than 0.05 were considered statistically significant. In the second step, the associations of patient characteristics with shunt insertion were analysed using relative risk ( $R R, 95 \% \mathrm{Cl})$, after which we ran various models adjusted by RR $(95 \% \mathrm{Cl})$ including variables found to be significant in the first step. Statistical analysis was also performed using forward stepwise logistic regression (cut-off value of $p=0.05$; Wald test) with the use of a shunt as the dependent variable, and the following as independent variables: high blood pressure (HBP); percentages of occlusion in ipsilateral disease and, separately, in contralateral disease; CVA; TIA; and asymptomatic status. Potential associations between preoperative and intraoperative variables and shunt were first tested by univariate analysis. We also calculated the relative excess risk due to interaction of the risk factors identified in the study [20].

Statistical calculations were performed using SPSS statistical software, v.15 (SPSS, Chicago, IL, USA).

\section{Results}

The cohort investigated $(n=80)$ comprised 71 men (88.75\%) and 9 women (11.25\%), who ranged from 45 to 82 years old (mean age: $66.06 \pm 9.07$ years; mean weight: $72.24 \pm 9.32 \mathrm{~kg}$ ). The mean degree of stenosis was $85.2 \pm 8.7 \%$, with a minimum and maximum stenosis of $70 \%$ and $99 \%$.

Previous symptoms were TIA including amaurosis fugax $(n=21)$ and CVA that included reversible ischaemic neurological deficits (RINDs) $(n=41)$ or both $(n=2)$. In 62 symptomatic patients $(77.5 \%)$ the degree of carotid stenosis was $86.7 \pm 8.6 \%$ and in the 18 asymptomatic patients (22.5\%) it was 80.5 $\pm 7.6 \%$, with a $p$ value $=0.019$.

The demographic data and medical history of shunted and non-shunted patients are given in Table I. Shunted patients had significantly higher levels of contralateral stenosis and they had a higher frequency of previous symptoms (described before) although this was not significant.

In 15 patients (18.5\%) clinical cerebral ischaemia was diagnosed by mental confusion, agitation, restlessness and/or contralateral upper limb motor deficit. In 11 of these patients $(11 / 15,73.3 \%)$ the deficit occurred at the time of the 3-min clamping test and it was reversed after shunt insertion. Overall, a shunt was needed in 12 patients (15.0\%), 11 around the time of clamping when the deficit 
Interaction of cerebrovascular disease and contralateral carotid occlusion in prediction of shunt insertion during carotid endarterectomy

Table I. Baseline preoperative characteristics of patients included in the study $(n=80)$

\begin{tabular}{|c|c|c|c|c|}
\hline \multirow[t]{2}{*}{ Parameter } & & \multicolumn{2}{|c|}{ Shunt } & \multirow[t]{2}{*}{ Value of $p^{*}$} \\
\hline & & $\begin{array}{l}\text { Shunted patients } \\
\qquad(n=12)\end{array}$ & $\begin{array}{l}\text { Non-shunted patients } \\
\qquad(n=68)\end{array}$ & \\
\hline \multirow[t]{5}{*}{ Age [years] } & Mean \pm SD & $64.4 \pm 7.9$ & $66.4 \pm 9.1$ & 0.49 \\
\hline & Min-max & $50-76$ & $45-82$ & \\
\hline & $\leq 65$ & $8(66.7 \%)$ & $28(41.2 \%)$ & 0.11 \\
\hline & $65-74$ & $3(25.0 \%)$ & $25(36.8 \%)$ & 0.65 \\
\hline & $\geq 75$ & $1(8.3 \%)$ & $15(22.1 \%)$ & 0.48 \\
\hline \multirow[t]{2}{*}{ Gender } & Male & $11(91.7 \%)$ & $60(88.2 \%)$ & 0.88 \\
\hline & Female & $1(8.3 \%)$ & 8 (11.8\%) & \\
\hline \multirow[t]{3}{*}{ ASA } & ॥ & $0(0.0 \%)$ & $8(11.9 \%)$ & \\
\hline & III & $9(75.0 \%)$ & 49 (73.1\%) & 0.80 \\
\hline & IV & $3(25.0 \%)$ & $10(14.9 \%)$ & 0.64 \\
\hline \multirow[t]{2}{*}{ Surgical carotid } & Left & $8(66.7 \%)$ & 37 (56.1\%) & 0.49 \\
\hline & Right & $4(33.3 \%)$ & $29(43.9 \%)$ & \\
\hline \multirow[t]{3}{*}{ Degree of stenosis [\%] } & Mean & $84.0+10.1$ & $85.4 \pm 8.5$ & 0.60 \\
\hline & $70-90 \%$ & $5(41.7 \%)$ & $33(50.0 \%)$ & 0.51 \\
\hline & $>90 \%$ & $7(58.3 \%)$ & $33(50.0 \%)$ & 0.59 \\
\hline \multirow[t]{4}{*}{ Contralateral stenosis [\%] } & Mean & $66.7 \pm 33.7$ & $31.3 \pm 33.1$ & $0.04^{*}$ \\
\hline & $\leq 50 \%$ & $2(16.7 \%)$ & $38(55.9 \%)$ & $0.01^{*}$ \\
\hline & $50-70 \%$ & $5(41.7 \%)$ & 19 (27.9\%) & 0.54 \\
\hline & $\geq 70 \%$ & $5(41.7 \%)$ & $11(16.2 \%)$ & 0.10 \\
\hline \multicolumn{5}{|c|}{ With previous symptoms: $62 / 80$ (77.5\%) } \\
\hline & TIA & $1(8.3 \%)$ & $22(32.4 \%)$ & 0.18 \\
\hline & CVA & $9(75.0 \%)$ & $32(47.1 \%)$ & 0.07 \\
\hline Asymptomatics: 18/80 (22.5\%) & & $2(16.7 \%)$ & $16(23.5 \%)$ & 0.88 \\
\hline HBP & $56(70.0 \%)$ & $10(83.3 \%)$ & $46(68.9 \%)$ & 0.51 \\
\hline Controlled HBP & & $9(75.0 \%)$ & $39(84.8 \%)$ & 0.64 \\
\hline \multirow[t]{6}{*}{$\mathrm{BP}$ at admission } & Mean \pm SD & & & \\
\hline & Min-max & & & \\
\hline & Systolic BP & $142.2 \pm 18.4$ & $141.2 \pm 15.9$ & 0.85 \\
\hline & & $130-180$ & $100-170$ & \\
\hline & Diastolic BP & $74.5 \pm 7.8$ & $75.3 \pm 11.2$ & 0.82 \\
\hline & & $80-55$ & $90-50$ & \\
\hline IHD & & $4(33.3 \%)$ & $13(21.0 \%)$ & 0.55 \\
\hline AMI treatment & & $3(75.0 \%)$ & $9(69.2 \%)$ & 0.95 \\
\hline \multirow[t]{2}{*}{ IHD progression in years } & Mean \pm SD & $6.9 \pm 3.1$ & $4.6 \pm 5.0$ & 0.15 \\
\hline & Min-max & $4-13$ & $1-18$ & \\
\hline Non-ischaemic heart disease & & 0 & $6(9.0 \%)$ & \\
\hline Peripheral artery disease & & $6 / 12(50.0 \%)$ & $30 / 68(44.1 \%)$ & 0.71 \\
\hline
\end{tabular}


Table I. cont.

\begin{tabular}{|c|c|c|c|c|}
\hline \multirow{2}{*}{\multicolumn{2}{|c|}{ Parameter }} & \multicolumn{2}{|c|}{ Shunt } & \multirow[t]{2}{*}{ Value of $p^{*}$} \\
\hline & & $\begin{array}{l}\text { Shunted patients } \\
\qquad(n=12)\end{array}$ & $\begin{array}{l}\text { Non-shunted patients } \\
\qquad(n=68)\end{array}$ & \\
\hline \multicolumn{2}{|c|}{ Antiplatelet drugs $62 / 80$ (77,5\%) } & $11(91.7 \%)$ & $51(75.0 \%)$ & 0.81 \\
\hline & Aspirin & $3(25.0 \%)$ & $17(25.4 \%)$ & 0.72 \\
\hline & Clopidogrel & $4(33.0 \%)$ & $24(35.8 \%)$ & 1 \\
\hline & Aspirin + clopidogrel & $4(33.0 \%)$ & $8(11.9 \%)$ & 0.14 \\
\hline & Acenocoumarol & 0 & $2(3.0 \%)$ & \\
\hline \multicolumn{2}{|l|}{ Renal disease } & 0 & $9(13.4 \%)$ & \\
\hline \multicolumn{2}{|l|}{ Dyslipidaemia 56 (70.0\%) } & $11(91.7 \%)$ & $45(67.2 \%)$ & 0.18 \\
\hline \multicolumn{2}{|l|}{ Statin therapy $43 / 56(76.8 \%)$} & $9(81.8 \%)$ & $34(75.6 \%)$ & 0.72 \\
\hline \multicolumn{2}{|l|}{ Smoking habit } & $11(91.6 \%)$ & $53(80.3 \%)$ & 0.62 \\
\hline \multicolumn{2}{|c|}{ NIDDM/IDDM 15(18.8\%)/10 (12.5\%) } & $1(8.3 \%) / 1(8.3 \%)$ & $14(20.9 \%) / 9(13.4 \%)$ & $0.55 / 1$ \\
\hline
\end{tabular}

Data are mean \pm standard deviation (SD) or number (\%) and minimum and maximum (min-max). TIA - transient ischaemic accident, CVA - cerebral vascular accident, HBP - high blood pressure, BP - blood pressure, IHD - ischaemic heart disease, AMI-acute myocardial infarction, NIDDM - non-insulin-dependent diabetes mellitus, IDDM - insulin-dependent diabetes mellitus

${ }^{*} p$ value $<0.05, \chi^{2}$ and analysis of variance (ANOVA) tests

appeared. In just 1 patient $(1 / 15,6.6 \%)$ the deficit occurred before clamping with restlessness and agitation [19] and anaesthesia was switched over to general prior to insertion of the shunt. The other three patients $(3 / 15,20 \%)$ presented neurological deficits after the clamping period. None of these shunted patients reported permanent neurological deficits on discharge from hospital.

Table II shows perioperative data and postoperative complications stratified according to whether or not patients had a shunt placed. There were no statistical differences between patients. Regarding neurological complications, two (2.5\%) of the non-shunted patients had CVAs after surgery while 1 patient suffered permanent deficit due to surgical carotid occlusion. Two non-shunted patients died 30 days after surgery: one from an intracranial haemorrhage, in the context of a hyperaemic response, and one from a ruptured aneurysm, while another non-shunted patient suffered from angina pectoris in the postoperative period. Eighteen patients (22.5\%) suffered from other complications including cervical haematoma and cranial nerve paralysis, with no significant differences. In none of these patients was discharge delayed.

In Table III, the RRs of shunted patients are shown, with respect to non-shunted patients. We observed that contralateral stenosis $\geq 50 \%$ (RR: 1.3; 95\% Cl: 1.0-1.5) and previous symptoms of CVA (RR: $1.2 ; 95 \% \mathrm{Cl}: 1.0-1.4)$ were associated with shunt insertion. With contralateral stenosis $\geq 50 \%$ and previous symptoms of CVA combined, the RR increased to 7.7 (95\% Cl: 1.0-14.4). The calculated relative excess risk due to interaction of contralateral stenosis $\geq 50 \%$ and previous symptoms of CVA was 6.2.

\section{Discussion}

The main finding of this study was the interaction existing in patients with contralateral stenosis $\geq 50 \%$ associated with previous CVA, which has not been previously reported. These patients have a seven-fold greater tendency to require shunt insertion in patients under regional anaesthesia. Impaired ischaemic tolerance after previous strokes has already been observed [7, 12-14]. In the present study there was also a higher shunt frequency in patients who had suffered a prior stroke $(83.3 \%$ vs. $76.6 \%$ ), but this difference did not reach statistical significance, probably due to the small sample size. The shunt incidence in the group with a contralateral ICA $\geq 50 \%$ was $83.4 \%$ vs. $43 . \%$. Shunted patients had a poorer health status than non-shunted patients in terms of pre-, intra- and postoperative parameters. It is likely that shunted patients did not have sufficient collateral cerebral circulation or that they had more cerebral areas at risk of ischaemia from changes in functional metabolism $[12,21]$, so were more sensitive to any kind of cerebral hypoperfusion. The reported results could support the view that the presence of unilateral, asymptomatic carotid stenosis is not associated with an increased risk of stroke following cardiac surgery if prophylactic CEA is not performed [22].

If we had followed the recommendation that carotid surgery can be performed safely without a shunt in patients with contralateral stenosis $\leq 50 \%$ and without previous ACV, we would have 
Table II. Perioperative data and postoperative complications

\begin{tabular}{|c|c|c|c|c|}
\hline \multirow[t]{2}{*}{ Parameter } & & \multicolumn{2}{|c|}{ Shunt } & \multirow[t]{2}{*}{ Value of $p^{*}$} \\
\hline & & $\begin{array}{l}\text { Shunted patients } \\
\qquad(n=12)\end{array}$ & $\begin{array}{l}\text { Non-shunted patients } \\
\qquad(n=68)\end{array}$ & \\
\hline \multirow[t]{2}{*}{ Duration of surgery [min] } & Mean \pm SD & $126.4 \pm 39.8$ & $108.3 \pm 21.9$ & 0.24 \\
\hline & Min-max & $75-210$ & $65-185$ & \\
\hline \multicolumn{5}{|l|}{ Duration of clamping [min] } \\
\hline & Mean \pm SD & $50.8 \pm 15.6$ & $43.9 \pm 16.5$ & 0.18 \\
\hline & Min-max & $30-70$ & $17-110$ & \\
\hline \multirow[t]{2}{*}{ ICU [days] } & Mean \pm SD & $1.2 \pm 0.7$ & $1.3 \pm 0.7$ & 0.86 \\
\hline & Min-max & $1-3$ & $1-4$ & \\
\hline \multirow[t]{2}{*}{ Hospital stay } & Mean \pm SD & $3.7 \pm 2.1$ & $3.6 \pm 1.1$ & 0.95 \\
\hline & Min-max & $2-9$ & $3-8$ & \\
\hline \multicolumn{5}{|l|}{ Complications } \\
\hline No complications & & $9(75.0 \%)$ & $48(70.6 \%)$ & 0.98 \\
\hline \multirow[t]{5}{*}{ With complications } & & $3(25.0 \%)$ & $20(29.4 \%)$ & 0.88 \\
\hline & Neurological & 0 & $2(2.9 \%)$ & \\
\hline & Postoperative AMI & 0 & $1(1.5 \%)$ & \\
\hline & 30 day death & 0 & $2(2.9 \%)$ & \\
\hline & Other complications & $3(25.0 \%)$ & $15(22.1 \%)$ & 0.88 \\
\hline \multicolumn{5}{|l|}{ Postoperative BP Mean \pm SD } \\
\hline \multirow[t]{5}{*}{ Systolic BP } & $1 \mathrm{~h}$ & $144.7 \pm 29.8$ & $133.94 \pm 27.2$ & 0.21 \\
\hline & $6 \mathrm{~h}$ & $136.2 \pm 28.8$ & $133.2 \pm 27.1$ & 0.73 \\
\hline & $12 \mathrm{~h}$ & $140.3 \pm 22.0$ & $127.4 \pm 21.9$ & 0.06 \\
\hline & $18 \mathrm{~h}$ & $148.1 \pm 19.3$ & $135.7 \pm 21.9$ & 0.07 \\
\hline & $24 \mathrm{~h}$ & $151.1 \pm 19.3$ & $138.1 \pm 24.3$ & 0.08 \\
\hline \multirow[t]{5}{*}{ Diastolic BP } & $1 \mathrm{~h}$ & $66.1 \pm 14.5$ & $62.9 \pm 14.1$ & 0.47 \\
\hline & $6 \mathrm{~h}$ & $62.2 \pm 14.9$ & $60.3 \pm 14.2$ & 0.67 \\
\hline & $12 \mathrm{~h}$ & $62.5 \pm 11.3$ & $57.7 \pm 12.4$ & 0.22 \\
\hline & $18 \mathrm{~h}$ & $65.6+10.3$ & 60.6 v12.6 & 0.20 \\
\hline & $24 \mathrm{~h}$ & $70.6 \pm 7.6$ & $68.0 \pm 14.7$ & 0.35 \\
\hline \multicolumn{2}{|c|}{ Intraoperative antihypertensive drugs } & $9 / 12(75.0 \%)$ & $38 / 68(55.9 \%)$ & 0.46 \\
\hline \multicolumn{2}{|c|}{ Postoperative antihypertensive drugs } & $6 / 11(54.5 \%)$ & $21 / 68(30.9 \%)$ & 0.13 \\
\hline
\end{tabular}

Data are mean \pm standard deviation (SD); BP-blood pressure, ICU - intensive care unit

put three patients at risk of developing major neurological deficits. Overall contralateral stenosis $\geq 50 \%$ and previous ACV wrongly predicted the requirement for a shunt in $36.8 \%$ (25/68), roughly a third of the patients. We found this a poor predictor relation with the awake neurological test.

Contrary to other reports where previous neurological symptomatology and/or intraoperative shunting were identified risk factors for increased complications [23], in our study there was not an increase of complications for patients with signifi- cant preoperative variables (contralateral stenosis $\geq 50 \%$ and previous symptoms of (VA) or intraoperative shunt insertion and postoperative high blood pressures were not significant. Recent reviews have highlighted the importance of optimal medical therapy with antiplatelet drugs and statins [24, 25]. Statins are effective in reducing both first-ever and recurrent stroke [26] and antiplatelet drugs are also very effective to avoid the platelet hyperaggregation state in CEA [27]. Statin effects may improve collateral blood flow, enhance cerebral 
Table III. Different models of relative risk for shunt use adjusted for previously identified patient characteristics

\begin{tabular}{|ll|}
\hline Patient characteristics & $\begin{array}{l}\text { Shunt } \\
\text { Relative risk }(95 \% \mathrm{Cl})\end{array}$ \\
\hline Contralateral stenosis $\geq 50.0 \%$ & $1.3(1.0-1.5)^{\star}$ \\
\hline Previous symptoms CVA_TIA & $0.9(0.8-1.2)$ \\
\hline Previous symptoms CVA & $1.2(1.0-1.4)^{*}$ \\
\hline Contralateral stenosis $\geq 50.0 \%$ and previous symptoms CVA_ TIA & $5.9(0.8-43.5)$ \\
\hline Contralateral stenosis $\geq 50.0 \%$ and previous symptoms CVA & $7.7(1.0-14.4)^{*}$ \\
\hline
\end{tabular}

CI-confidence interval, TIA - transient ischaemic accident, CVA - cerebral vascular accident; *significant interval, Wald test, $p<0.05$

vasodilation during periods of compromised flow, and prevent carotid thrombosis and embolism in the perioperative period. Both antiplatelet drugs and statins were used in $91.75 \%$ of shunted patients and $75-67.2 \%$ respectively of the nonshunted patients. The use of shunt and optimal medical preventative therapies should be complementary: shunt insertion would be usefully supported by antiplatelet therapy, to avoid associated thromboembolic complications, while the shunt would help to prevent stroke by hypoperfusion.

Selective shunting has been related to postoperative cognitive dysfunction [4]. Better cognitive outcomes have been observed following CEA under regional anaesthesia [28] and, in addition, cardiac and pulmonary morbidity associated with general anaesthesia can be avoided. While this approach involves more stress for the medical professionals involved, it seems to offer significant benefits to patients. The GALA study [29] compared general versus regional anaesthesia in 3526 patients. In relation to major complications of stroke, $A M I$ or death, there was no evidence for deciding whether to use general or regional anaesthesia. However, regional anaesthesia did decrease shunt insertion (14\% vs. $43 \%, p<0.001)$. The need to increase blood pressure was more common under general anaesthesia, while it was more often necessary to decrease blood pressure when regional anaesthesia was used. In other research, higher levels of postoperative hypertension have been attributed to pharmacological or surgical carotid sinus denervation [30]. Our patients also mainly needed antihypertensive drugs and this was most common in shunted patients in the intraoperative and in the postoperative period, especially $12 \mathrm{~h}$ post surgery (Table II). Hypertension is the most important risk factor for stroke [31] and reperfusion syndrome; for this reason it is advisable to monitor these shunted patients in the ICU during the immediate postoperative period. In the GALA study, rates of reversible cranial nerve lesions, haematoma and reintervention did not differ between the types of anaesthesia, in spite of the lower rates of shunt use in regional anaesthesia. In our study, there were more of these complications in shunted patients, which we attributed to the arterial surgical manipulation, but the difference was not significant.

The limitations of this study are that a relatively low percentage of patients were shunted and had serious complications, and that the sample size is not sufficiently large to draw strong conclusions. However, this study has a high level of internal validity and homogeneity because it was carried out under a strict protocol and by the same team, preventing treatment protocol bias, which is not the case in other studies involving hundreds of patients from different hospitals and under different protocols.

Our findings suggest that patients with contralateral occlusion $\geq 50 \%$ and with previous CVA present greater risks of shunt use during CEA than patients with these risk factors separately. The combination of both contralateral occlusion $\geq 50 \%$ and previous CVA become synergic and strengthen the risk of shunt insertion.

\section{References}

1. Brott TG, Hobson RW, Howard G, et al. Stenting versus endarterectomy for treatment of carotid-artery stenosis. N Engl J Med 2010; 363: 11-23.

2. Rudarakanchana N, Dialynas M, Halliday A. Asymptomatic Carotid Surgery Trial-2 (ACST-2): rationale for a randomised clinical trial comparing carotid endarterectomy with carotid artery stenting in patients with asymptomatic carotid artery stenosis. Eur J Vasc Endovasc Surg 2009; 38: 239-42.

3. Bond R. Derivacion (shunt) sistematica o selectiva de la arteria carotida para la endarterectomia carotidea (y otros metodos de control en la derivacion selectiva) (Revision Cochrane traducida). La biblioteca cochrane plus, 2008 Número 2 Oxford: Update Software Ltd Disponibe en: http://www update-sofware com (Traducida de The Cochrane Library, 2008 Issue 2 Chichester, UK: John Wiley \& Sons, Ltd) 2010.

4. Hudorovic N, Lovricevic I, Hajnic H, Ahel Z. Postoperative internal carotid artery restenosis after local anesthesia: presence of risk factors versus intraoperative shunt. Interact Cardiovasc Thorac Surg 2010; 11: 182-4.

5. Mazul-Sunko B, Hromatko I, Tadinac M, et al. Subclinical neurocognitive dysfunction after carotid endarterectomy- 
the impact of shunting. J Neurosurg Anesthesiol 2010; 22: 195-201.

6. Estruch-Perez MJ, Barbera-Alacreu M, Usina-Aguilar A, et al. Bispectral index variations in patients with neurological deficits during awake carotid endarterectomy. Eur J Anaesthesiol 2010; 27: 359-63.

7. Tan TW, Garcia-Toca M, Marcaccio EJ Jr, et al. Predictors of shunt during carotid endarterectomy with routine electroencephalography monitoring. J Vasc Surg 2009; 49: $1374-8$

8. Kim MS, Cole DJ. Anesthesia for carotid surgery. ASA Refresher courser 2003; 31: 91-103.

9. Norris EJ. Anesthesia for vascular surgery. Miller's Anesthesi 2005; 2098-105.

10. Rothwell PM, Slattery J, Warlow CP. A systematic comparison of the risks of stroke and death due to carotid endarterectomy for symptomatic and asymptomatic stenosis. Stroke 1996; 27: 266-9.

11. Blanco Cañibano E, Reina Gutiérrez T, Serrano Hernando F, et al. Endarterectomía carotídea con anestesia locorregional. Estudio de factores clínicos y arteriográficos de riesgo de isquemia cerebral durante el clampaje carotídeo. Angiologia 2004; 56: 133-45.

12. Schneider JR, Droste JS, Schindler N, et al. Carotid endarterectomy with routine electroencephalography and selective shunting: influence of contralateral internal carotid artery occlusion and utility in prevention of perioperative strokes. J Vasc Surg 2002; 35: 1114-22.

13. Jacobowitz GR, Rockman CB, Lamparello PJ, et al. Causes of perioperative stroke after carotid endarterectomy: special considerations in symptomatic patients. Ann Vasc Surg 2001; 15: 19-24.

14. Aleksic M, Gawenda M, Heckenkamp J, et al. Prediction of cerebral ischemic tolerance during carotid crossclamping by angiographic criteria. Eur J Vasc Endovasc Surg 2004; 27: 640-5.

15. Ballotta E, Saladini M, Gruppo M, et al. Predictors of electroencephalographic changes needing shunting during carotid endarterectomy. Ann Vasc Surg 2010; 24: 1045-52.

16. Grant EG, Benson CB, Moneta GL, et al. Carotid artery stenosis: gray-scale and Doppler US diagnosis: Society of Radiologists in Ultrasound Consensus Conference. Radiology 2003; 229: 340-6.

17. Winnie AP, Ramamurthy S, Durrani Z, Radonjic R. Interscalene cervical plexus block: a single-injection technic. Anesth Analg 1975; 54: 370-5.

18. Moore DC. Regional block: a handbook for use in the clinical practice of medicine and surgery. 4th Ed. Springfield, Illinois 1978.

19. Stoneham MD, Knighton JD. Regional anaesthesia for carotid endarterectomy. Br J Anaesth 1999; 82: 910-9.

20. Greenland S, Lash TL, Rothman KJ. Concepts of interaction. In: Modern epidemiology. Sonya Seigafuse (ed.). Lippincott, Williams and Wilkins, Philadelphia 2008; 71-86.

21. Sanchez-Chavez JJ. The penumbra area. Rev Neurol 1999; 28: 810-6.

22. Baiou D, Karageorge A, Spyt T, Naylor AR. Patients undergoing cardiac surgery with asymptomatic unilateral carotid stenoses have a low risk of peri-operative stroke. Eur J Vasc Endovasc Surg 2009; 38: 556-9.

23. Aleksic M, Luebke T, Brunkwall J. Outcome of carotid endarterectomy under local anaesthesia with respect to the patients' risk profile. Vasa 2009; 38: 225-33.

24. Maharaj R. A review of recent developments in the management of carotid artery stenosis. J Cardiothorac Vasc Anesth 2008; 22: 277-89.

25. Sillesen $\mathrm{H}$. What does 'best medical therapy' really mean? Eur J Vasc Endovasc Surg 2008; 35: 139-44.
26. Amarenco P, Lavallée PC, Mazighi M, Labreuche J. The role of statins in the prevention of stroke. Arch Med Sci 2007; 3: S109-14.

27. Morozov I, Charnaia MA, Gladysheva VG. The causes of early disorders of cerebral circulation in patients undergoing carotid endarterectomy. Angiol Sosud Khir 2006; 12: 21-7.

28. Weber CF, Friedl H, Hueppe M, et al. Impact of general versus local anesthesia on early postoperative cognitive dysfunction following carotid endarterectomy: GALA Study Subgroup Analysis. World J Surg 2009; 33: 1526-32.

29. Lewis SC, Warlow CP, Bodenham AR, et al. General anaesthesia versus local anaesthesia for carotid surgery (GALA): a multicentre, randomised controlled trial. Lancet 2008; 372: 2132-42.

30. Al-Rawi PG, Sigaudo-Roussel D, Gaunt ME. Effect of lignocaine injection in carotid sinus on baroreceptor sensitivity during carotid endarterectomy. J Vasc Surg 2004; 39: 1288-94.

31. Sierra C, Domenec M, Coca A. Brain damage in hypertension. Arch Med Sci 2009; 5: S254-66. 